\title{
Monitoring of Cocrystal Dissociation during the Wet Granulation Process in the Presence of Disintegrants by Using Low-Frequency Raman Spectroscopy
}

\author{
Naoto Suzuki, ${ }^{a}$ Kanako Fukui, ${ }^{b}$ Koki Otaka, ${ }^{b}$ Toyofumi Suzuki, ${ }^{a}$ and Toshiro Fukami*,b \\ ${ }^{a}$ Laboratory of Pharmaceutics, School of Pharmacy, Nihon University; Funabashi, Chiba 274-8555, Japan: and \\ ${ }^{b}$ Department of Molecular Pharmaceutics, Meiji Pharmaceutical University; Kiyose, Tokyo 204-8588, Japan. \\ Received April 9, 2021; accepted June 3, 2021
}

The aim of this study was to evaluate the effect of three coformers and five disintegrants in the granulation formulation on the dissociation of cocrystal during the granulation process by monitoring wet granulation with probe-type low-frequency Raman (LF-Raman) spectroscopy. As model cocrystals, paracetamol (APAP)-oxalic acid (OXA), APAP-maleic acid (MLA), and APAP-trimethylglycine (TMG) were used. The monitoring of the granulation recipe containing cocrystals during wet granulation was performed over time with high-performance LF-Raman spectrometry and the dissociation rate was calculated from the results of multivariate analysis of LF-Raman spectra. The dissociation rate decreased in the order of APAP-TMG, APAP-OXA, and APAP-MLA, showing the same order as observed in Powder X-ray diffraction measurements. Furthermore, to compare the effect of disintegrants on the dissociation rate of APAP-OXA, LFRaman monitoring was performed for the granulation recipes containing five typical disintegrants (two lowsubstitution hydroxypropyl cellulose (HPC), cornstarch (CSW), carmellose sodium (CMC), and crospovidone (CRP)). The dissociation rate of APAP-OXA decreased in the order of CSW, HPCs, CMC, and CRP. This difference in the dissociation rate of APAP-OXA was thought to be due to the disintegration mechanism of the disintegrants and the water absorption ratio, which was expected to affect the water behavior on the disintegrant surface during wet granulation. These results suggested that probe-type LF-Raman spectroscopy is useful to monitor the dissociation behavior of cocrystals during wet granulation and can compare the relative stability of cocrystal during wet granulation between different formulations.

Key words cocrystal; dissociation; low-frequency Raman; disintegrant; wet granulation

\section{Introduction}

Pharmaceutical cocrystals are crystalline complexes consisting of active pharmaceutical ingredients (APIs) and coformers such as excipients that interact through non-covalent bonds. Cocrystals are applied not only to neutral compounds without dissociable groups but also to acidic or basic compounds with dissociable groups. Additionally, cocrystallization of APIs has attracted attention in the past decade as a common strategy to improve physicochemical properties, such as solubility, stability, dissolution rate, and bioavailability, without altering their chemical covalent structure and inherent pharmacological activities. ${ }^{1,2)}$ Due to these advantages, cocrystals have been mentioned as an equivalent solid-state form to salt in the revised guidance issued by the Food and Drug Administration (FDA) ${ }^{3)}$ and the reflection paper published by the European Medicines Agency. ${ }^{4)}$ Pharmaceutical products containing cocrystals have been marketed, e.g., Depakote (valproic acid-sodium valproate, AbbVie), Lexapro (escitalopram oxalate-oxalic acid, Mochida), Entresto (valsartan-sacubitril, Novartis), ${ }^{5)}$ Steglatro (ertugliflozin-L-pyroglutamic acid, MERCK), and Suglat (ipraglifozin-L-proline, Astellas). ${ }^{6}$

In the past, cocrystal researchers focused on the screening of novel cocrystals and their physicochemical and pharmacokinetic evaluation. However, these researchers have recently worked toward preparing cocrystals during mechanical or thermal loading manufacturing processes such as hot-melt extrusion, shear milling technology, and fluidized bed granulation. $^{7-9)}$ This trend has since been referred to as continu- ous manufacturing, in which the materials and products are continuously charged into and discharged from the system throughout the process, and is attracting attention to reduce the manufacturing cost, shorten the time to market and produce high-quality pharmaceuticals. ${ }^{10,11)}$ In 2019, the FDA issued draft guidance related to continuous manufacturing of solid dosage forms, ${ }^{12)}$ and it is expected that pharmaceutical products consisting of crystalline complexes such as cocrystals prepared by continuous manufacturing will be marketed frequently in the future. In this context, there has been an increasing interest in monitoring the solid-state form, which affects the final product quality, during the manufacturing process. Thus, the improvement and installation of process analytical technology (PAT), which enables real-time analysis at the pharmaceutical manufacturing site, has evolved dramatically.

Raman spectroscopy and near-infrared spectroscopy have been reported as representative PAT tools. ${ }^{13-16)}$ In particular, Raman spectroscopy is a nondestructive, noncontact, and sample pretreatment-free method for characterizing molecular states. ${ }^{17,18)}$ The advantages of this technique over other PAT tools are that the water interference is minimal, ${ }^{19,20)}$ and high molecular additives have no effect on the Raman spectra. ${ }^{21}$ Recently, the development of a notch filter capable of removing Rayleigh-scattered light has made it possible to measure Raman spectra in the low-frequency region $\left(10-200 \mathrm{~nm}^{-1}\right)$. The Raman spectrum change in the low-frequency region reflects the vibrational modes related to long-range order (i.e., 
crystallinity) that can provide unique information on the solid/ structure characteristics compared to the conventional region, which provides molecular state information due to the bond vibration between atoms. ${ }^{22}$ Therefore, low-frequency Raman spectroscopy (LF-Raman) can identify polymorphs ${ }^{23,24)}$ and cocrystals $^{25-27)}$ so that our group has also reported monitoring the solvent-mediated transition of carbamazepine by probe-type LF-Raman ${ }^{28)}$ and cocrystal formation by a reaction crystallization method using LF-Raman. ${ }^{29)}$ Additionally, understanding the dissociation phenomenon of cocrystals in the manufacturing process is essential to produce high-quality pharmaceutical products containing cocrystals. Kaur et al. ${ }^{30)}$ evaluated the effect of water and heat on the dissociation of cocrystals by powder X-ray diffraction measurements to predict the dissociation of crystalline complexes during manufacturing. They suggested that water adsorbed on the surface and mechanical loading due to grinding were the factors causing lattice disorder that triggered cocrystal dissociation. Duggirala et $a{ }^{31)}$ reported that when tablets containing caffeine-oxalic acid (OXA) cocrystals were stored under humidified conditions, the cocrystal dissociated due to water adsorption on the surface. For these reports, Nomura et $a l^{32)}$ succeeded in monitoring cocrystal dissociation in wet granulation, a unit operation, using LF-Raman. ${ }^{33)}$ However, it is hard to say that the LF-Raman system Nomura et al. ${ }^{32)}$ used could monitor cocrystal dissociation in real time because it took $5 \mathrm{~min}$ to obtain an LF-Raman spectrum. Furthermore, no reports have evaluated the effect of coformers and disintegrants, which are used in solid dosages as typical additives, on the dissociation of cocrystals in the manufacturing process to select the optimized solid-state form. To select the most stable cocrystal in the manufacturing process, it is essential to monitor the solid state in the manufacturing process using a PAT tool with high sensitivity and fast measurement.

In the present study, to compare the relative stability of crystalline complexes composed of paracetamol (APAP), the dissociation behavior of three cocrystals and one amide salt during wet granulation was monitored by LF-Raman measurements. The obtained Raman spectra were subjected to multivariate analysis to calculate the dissociation rate of each crystalline complexes. The stability of the crystalline complexes was compared by comparing the dissociation rates of each crystalline complexes. Furthermore, the effect of disintegrant on the stability of cocrystal in wet granulation was investigated by comparing the dissociation rate of APAP-OXA cocrystal, when several disintegrants with different disintegration mechanisms, which are expected to affect the moisture behavior in the formulation, were added.

\section{Experimental}

Materials We used APAP, which can form some co- crystals, as a model drug and maleic acid (MLA) (Tokyo Chemical Industry Co., Ltd., Tokyo, Japan; TCI), OXA, and trimethylglycine (TMG) (FUJIFILM Wako Pure Chemical Corporation, Osaka, Japan; WAKO) (Fig. S1). The following excipients were used in the granulation process: microcrystalline cellulose (MCC; Asahi Kasei Corp., Tokyo, Japan) and hydroxypropyl cellulose 6.0-10.0 (HPC: Wako). Furthermore, five disintegrants added to the granulation recipe are summarized in Table 1.

Preparation of Crystalline Complexes Containing APAP Cocrystals were prepared through the solvent evaporation method. Equimolar mixtures of APAP and each coformer were dissolved in solvents (OXA in acetonitrile, MLA in ethanol, and $\mathrm{TMG}$ in acetone/methanol $=2: 1)$, and these solvents were evaporated at $40^{\circ} \mathrm{C}$. Additionally, APAP- $p$-toluenesulfonic acid (TOSA) were prepared by the precipitation method. APAP and TOSA were solve in methanol and precipitated after 2-3d. These residual powders and precipitated, which were cocrystals and amide salt, were sieved through a $250 \mu \mathrm{m}$ mesh and applied to the following tests.

Monitoring of Cocrystal Dissociation Using Powder X-Ray Diffraction (PXRD) Five milligrams of physical mixture of equal amounts of each cocrystal and the disintegrants listed in Table 1 were mounted on a nonreflective silicone sample plate. Then, the crystallinity of the mixtures was evaluated by PXRD after a predetermined amount of water was put on the mixtures. PXRD measurements were performed using a MiniFlex 600 X-ray generator (Rigaku Corp., Tokyo, Japan). Collecting the PXRD patterns was performed at a voltage of $30 \mathrm{kV}$, a current of $15 \mathrm{~mA}$, scanning angles in the range of $5.0-35.0^{\circ}$, and a scan speed of $20^{\circ} \mathrm{min}^{-1}$ using a $\mathrm{Cu}-K \alpha$ radiation source.

LF Raman Spectroscopy Raman spectra were obtained during wet granulation in a high mixer granulator using a THz-Raman Probe system ${ }^{\circledR}$ (TR-Probe, excitation laser wavelength $=808 \mathrm{~nm}, 300 \mathrm{~mW}$ as laser source, notch filters; Coherent Inc., Monrovia, CA, U.S.A.) with a BallProbe ${ }^{\circledR}$ (diameter $=1 / 2$ inch, length $=10$ inches, focal length $=5 \mathrm{~mm}$ ) attached to an all-in-one Raman spectrometer (Marqmetrix, Seattle, WA, U.S.A.). The system was operated with MVVM software (Marqmetrix), and a spectrum was collected every minute at a $4 \mathrm{~cm}^{-1}$ resolution with 5 scans in the Raman shift range between 10 and $200 \mathrm{~cm}^{-1}$. Pure cocrystal spectra and dissociation profiles of the species involved in the monitored wet granulation process were calculated using the multivariate curve resolution (MCR) installed in HoloReact software (Kaiser Optical Systems Inc. Ann Arbor, MI, U.S.A.).

Granulating Process A schematic diagram of granulation is shown in Fig. 1. Initially, cocrystals, disintegrants (shown in Table 1), HPC, and MCC were premixed in portions of 5, 5,3 , and $87 \%$, respectively (only in the case of APAP-TOSA

Table 1. List of Disintegrants Used in the Monitoring of the Granulating Process

\begin{tabular}{lccc}
\hline \hline Disintegrants & Products & Abbreviation & Disintegration mechanism \\
\hline Crospovidone & Kollidone $^{\circledR}$ CL-F & CRP & Wicking \\
Low-substituted hydroxypropyl cellulose & L-HPC ${ }^{\circledR}$ LH-21 & HPCw & Swelling \\
& L-HPC $^{\mathbb{B}}$ NBD-021 & HPCs & Swelling \\
Carmellose sodium & KICCOLATE $^{\mathrm{TM}}$ ND-2HS & CMC & Swelling \\
Cornstarch & Cornstarch W & CSW & Swelling \\
\hline
\end{tabular}




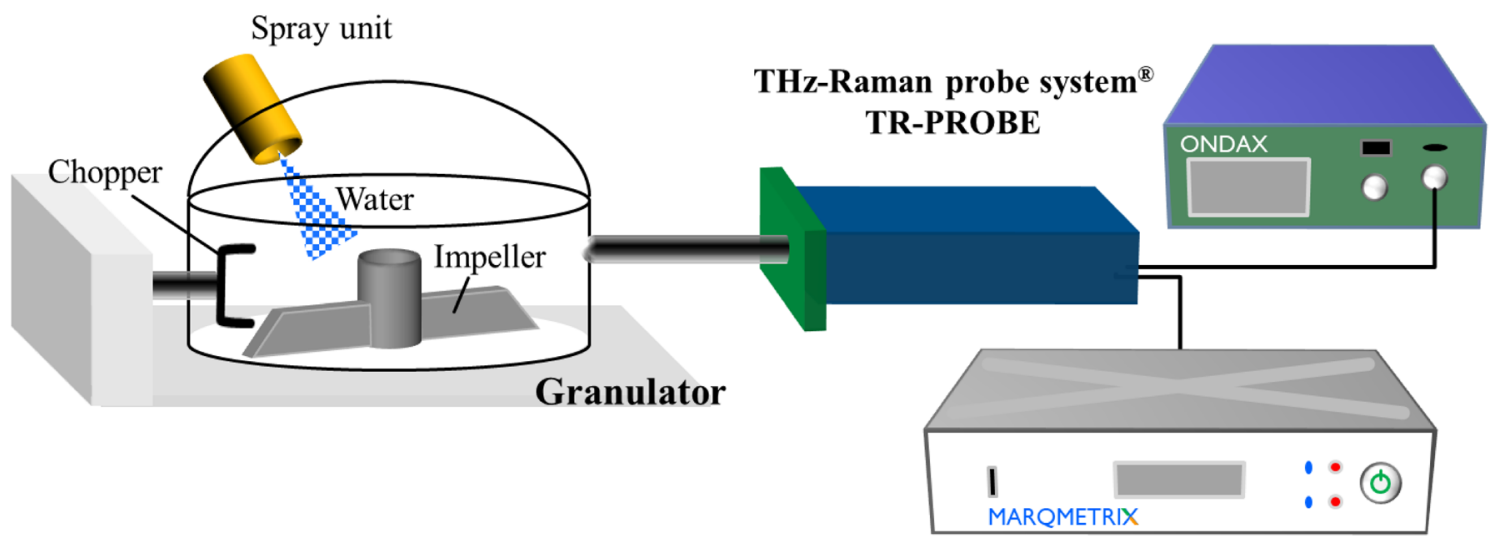

All-in-one Raman spectrometer

Fig. 1. Apparatus for in Situ Monitoring of the Wet Granulation Process Using a Low-Frequency Raman Spectrometer (Color figure can be accessed in the online version.)
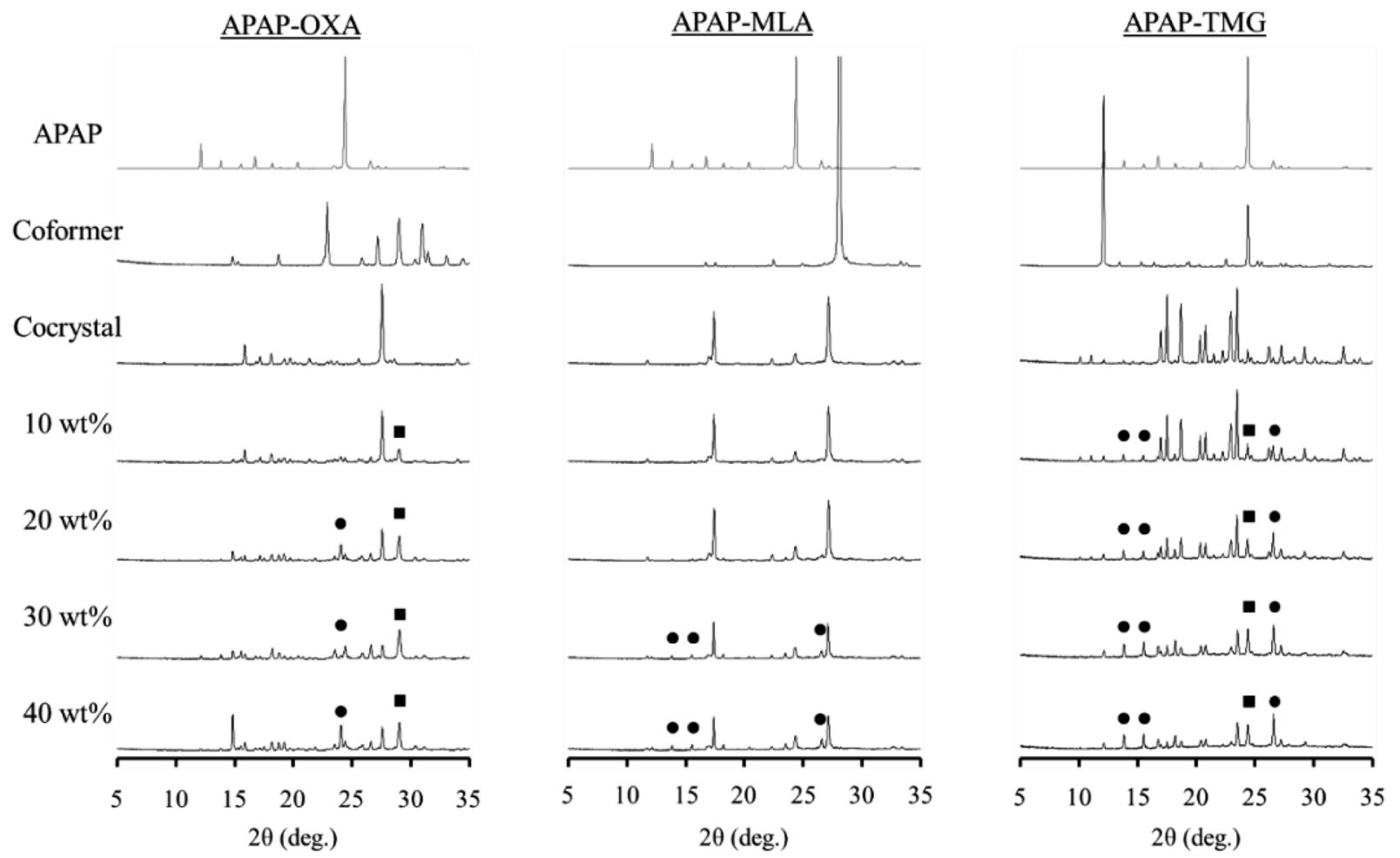

Fig. 2. Overlay of the APAP and Coformers and the Change in Diffraction Patterns Derived from Cocrystal Dissociation Caused by the Addition of Different Water Amounts

The newly generated APAP and coformer-derived diffraction peaks are indicated by

and $\mathbf{\square}$, respectively.

were the portions of APAP-TOSA and MCC 10 and $82 \%$, respectively). The premixtures were placed in a small acrylic high-shear granulator (Yoshiharaichiro Technical Professional Office, Tokyo, Japan). The granulation of this mixture proceeded by spraying the mixture with water at a rate of $1 \mathrm{~mL} / 3 \mathrm{~min}$. The impeller and chopper speeds were set at 300 and $1000 \mathrm{rpm}$, respectively. The amount of water added by 15 min of granulation was $5 \%$ of the solid component. The THz-Raman ${ }^{\circledR}$ probe was positionally fixed on the outer wall (through the granulator's acrylic wall) and used to obtain real time measurements of the crystalline state under noncontact and nondestructive conditions. The granulator's acrylic wall has no effect on the LF-Raman. ${ }^{32,33)}$ Polytetrafluoroethylene
(PTFE) tape (5490; 3 M Japan Limited, Tokyo, Japan) was attached to the spray unit outlet to prevent powder leakage. The exposure time and integration time were measured at $3 \mathrm{~s}$ and 5 acc, respectively, and the measurement interval was set to $1 \mathrm{~min}$.

Calculation of Cocrystal Dissociation Rate To evaluate the dissociation rate of the cocrystal during granulation, the fitting curve of the concentration profile of the cocrystal obtained from MCR analysis was used. For this evaluation, data from the start of granulation to $15 \mathrm{~min}$ were used. The slopes of the fitting curve resulting from the above analysis were calculated as the dissociation rate of each cocrystal to compare cocrystal stability. 
a)

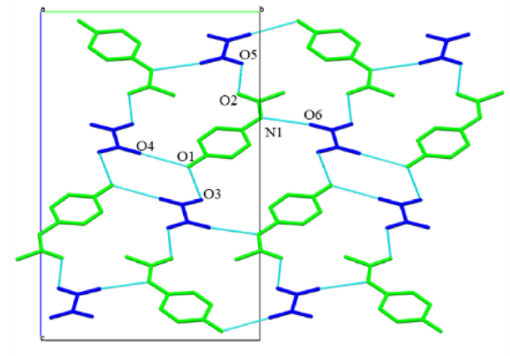

b)

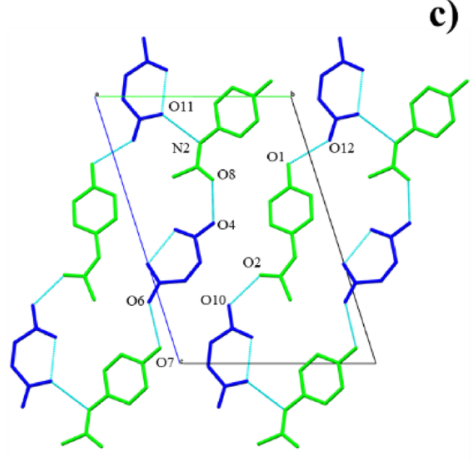

c)

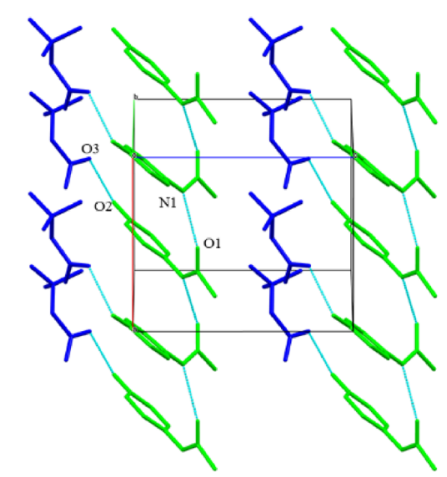

Fig. 3. Hydrogen Bonds as Intermolecular Interactions between APAP and Coformers in the Crystal Structures of a) APAP-OXA, b) APAP-MLA, and c) APAP-TMG

Green symbol: APAP molecule, blue symbol: conformer molecule. (Color figure can be accessed in the online version.)

Table 2. List of Hydrogen Bonds as Intermolecular Interactions between APAP and Coformers, Crystal Density and Water Solubility of Coformer

\begin{tabular}{|c|c|c|c|c|c|}
\hline & APAP & Coformer & Distance $(\AA)$ & Density $\left(\mathrm{g} / \mathrm{cm}^{3}\right)$ & Water solubility of coformer $(\mathrm{g} / 100 \mathrm{~mL})$ \\
\hline \multirow{4}{*}{ OXA } & N1 & O6 & 2.799 & \multirow{4}{*}{1.507} & \multirow{4}{*}{22.0} \\
\hline & $\mathrm{O} 1$ & $\mathrm{O} 3$ & 2.711 & & \\
\hline & $\mathrm{O} 1$ & $\mathrm{O} 4$ & 2.726 & & \\
\hline & $\mathrm{O} 2$ & O5 & 2.520 & & \\
\hline \multirow{6}{*}{ MLA } & $\mathrm{N} 2$ & $\mathrm{O} 11$ & 3.053 & \multirow{6}{*}{1.490} & \multirow{6}{*}{44.1} \\
\hline & $\mathrm{O} 1$ & $\mathrm{O} 12$ & 2.763 & & \\
\hline & $\mathrm{O} 2$ & $\mathrm{O} 10$ & 2.505 & & \\
\hline & $\mathrm{O} 7$ & O6 & 2.719 & & \\
\hline & O8 & $\mathrm{O} 4$ & 2.532 & & \\
\hline & - & $\mathrm{O} 4, \mathrm{O} 4$ & 3.014 & & \\
\hline \multirow{2}{*}{ TMG } & $\mathrm{N} 1, \mathrm{O} 1$ & - & 2.880 & \multirow{2}{*}{1.303} & \multirow{2}{*}{61.1} \\
\hline & $\mathrm{O} 2$ & $\mathrm{O} 3$ & 2.635 & & \\
\hline
\end{tabular}

\section{Results and Discussion}

Screening of Cocrystal Stability upon Water Addition by Using PXRD In the design of cocrystals, the difference in conformer affects the physicochemical properties of the formed cocrystal, such as solubility or stability. ${ }^{34)}$ In particular, the low stability of cocrystals during the manufacturing process can significantly impact the quality of the final products, including storage stability and bioavailability in vivo. Therefore, it is thought that measuring the stability of cocrystals in the manufacturing process is a crucial evaluation for selecting the optimal conformer or excipients.

To predict the stability of three cocrystals consisting of APAP, the crystallinity of the cocrystals immediately after adding different amounts of water was briefly screened by PXRD (Fig. 2). The intensity of the peaks derived from cocrystals decreased, and diffraction peaks derived from APAP $\left(13.8,15.6,24.03\right.$, and $\left.26.6^{\circ}\right)$, OXA dihydrate $\left(29.08^{\circ}\right)$, and TMG $\left(24.42^{\circ}\right)$ appeared when $10 \%$ water was added to APAPOXA and APAP-TMG. This change in diffraction patterns suggested that APAP-OXA and APAP-TMG partially dissociated by the addition of $10 \%$ water. On the other hand, when water was added to APAP-MLA, no change in peak intensity was observed up to $20 \%$ water, the intensity of peaks derived from cocrystals decreased, and the APAP-derived peaks appeared only at $30 \%$, suggesting that APAP-MLA partially dissociated by $30 \%$ water. These results indicated that APAPMLA was the most stable in the presence of water among the three cocrystals, while the stability of APAP-OXA and APAPTMG was almost equal and lower than that of APAP-MLA.

The discuss above results, the types of intermolecular interactions in the crystal structure of each cocrystal, the crystal density, the solubility of conformer in water were summarized in Fig. 3 and Table 2. As a result, the type of intermolecular interactions in the cocrystal structure were APAP-MLA, APAP-OXA and APAP-TMG in the order, consistent with the order of relative stability evaluated by PXRD. On the other hand, the crystal density of APAP-OXA and APAP-MLA were similar, and that of APAP-TMG was the lowest, and the solubility of coformer in water was in the order of TMG, MLA, OXA, which was different from the order of dissociability. In general, if one of the components of cocrystal has a good solubility in water, it will form an interaction with water when water was added, and the cocrystal will dissociate easily. Indeed, Maeno et al. $^{35)}$ also reported that the dissolution rate of APAP-TMG was faster than that of APAP or APAP-OXA. However, we previously revealed that APAPMLA formed when the physical mixture of APAP and MLA was stored at $40{ }^{\circ} \mathrm{C}$ and $75 \%$ relative humidity $(\mathrm{RH})$, whereas in the physical mixture of APAP and OXA, APAP-OXA did not form, and thermodynamically stable OXA hydrate appeared. ${ }^{36)}$ Additionally, in this study, when the same amount of water was added, cocrystal dissociation and OXA hydrate formation occurred in APAP-OXA, while cocrystals were maintained in APAP-MLA. This suggested that OXA hydrate 
a)

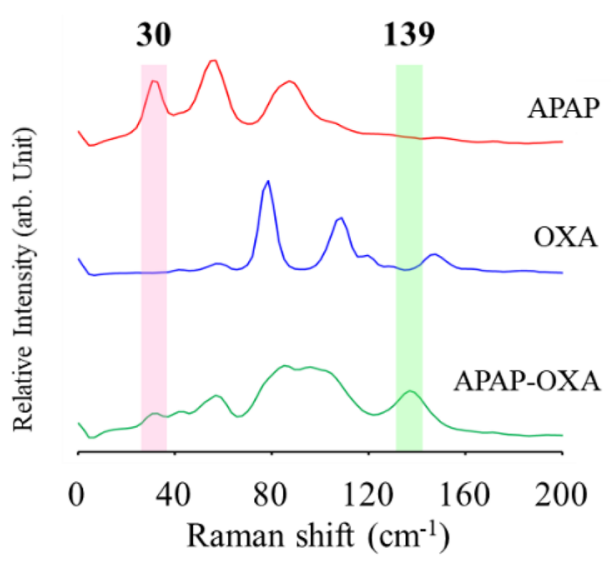

c)

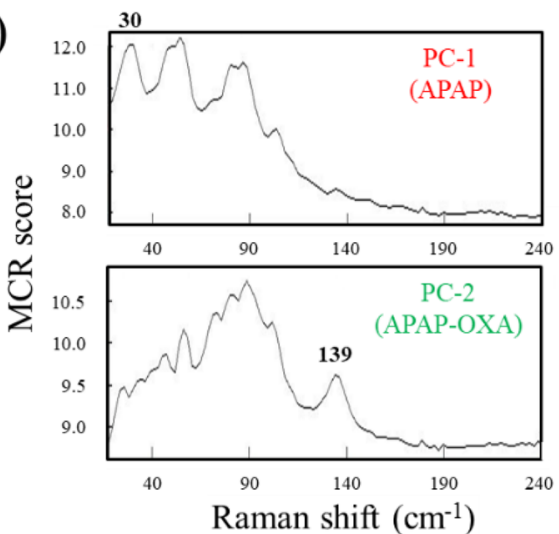

b)

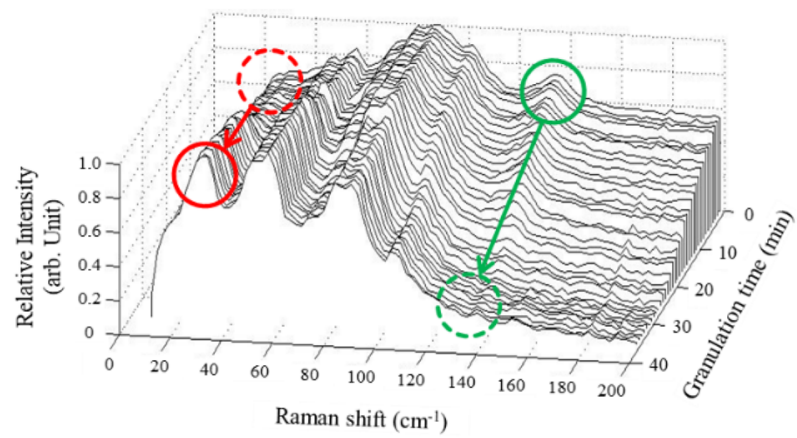

d)

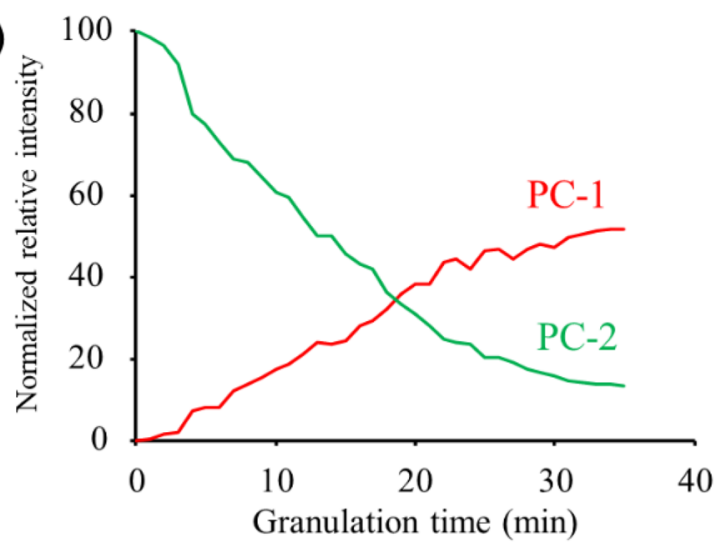

Fig. 4. a) Low-Frequency Raman Spectra of APAP, OXA, and APAP-OXA, b) the Waterfall Plot of Low-Frequency Raman Spectra of APAP-OXA during Wet Granulation, c) Principal Components from MCR Analysis, and d) Changes in the Relative Intensities of PC-1 (APAP) and PC-2 (APAPOXA) during Granulating Processes by Multivariate Curve Resolution Analysis

(Color figure can be accessed in the online version.)

is more energetically stable than APAP-OXA in the presence of water. Therefore, when the stability of APAP-OXA and APAP-MLA were compared, APAP-MLA considered to be more stable than APAP-OXA, since the formation of OXA hydrate was the driving force for the dissociation of APAPOXA, regardless of the crystal density or the solubility of the coformer in water. Thus, the stability of the three cocrystals was considered to depend on the number of hydrogen bonds between components and the stability of the crystals caused after the dissociation of the cocrystals. Therefore, PXRD measurements showed that it was possible to compare the stability of cocrystals containing APAP to water.

LF-Raman Monitoring of APAP-OXA Cocrystals Dissociated by Wet Granulation Our group previously reported that LF-Raman measurement over time can monitor cocrystal dissociation behavior in wet granulation. However, it is difficult to say that the monitoring of the solid-state form was conducted in real-time because it took $5 \mathrm{~min}$ to acquire one Raman spectrum. Additionally, the cocrystal content in the reported study was as high as $35 \%$, and detection in formulations with a low cocrystal content is essential for general use in various tablet formulations. In this study, we investigated whether it is possible to monitor the dissociation of cocrystals in wet granulation using the all-in-one Raman system (MarqMetrix, Inc.) equipped with a high laser power and high-sensitivity probe and can obtain spectra with an excellent signal-to-noise $(\mathrm{S} / \mathrm{N})$ ratio.

First, LF-Raman measurements of APAP, OXA, and APAPOXA in the low-frequency region showed characteristic peaks at $30 \mathrm{~cm}^{-1}$ and $139 \mathrm{~nm}^{-1}$ for APAP and APAP-OXA, respectively (Fig. 4a). In this region, no characteristic peaks were derived from excipients such as the binder, disintegrants, and the excipient in the premix, and the Raman intensities of these excipients were negligibly weak (Fig. S2). Although the excipients used in this study did not show Raman peaks in the low-frequency region, peaks appeared in this region when lactose and mannitol, which are crystalline excipients, were used as additives. ${ }^{15)}$ Therefore, when monitoring formulations containing these excipients, it is necessary to confirm the overlap between the peaks of the measured substance and the crystalline excipients in the low-frequency region in advance. The LF-Raman spectra of the granulation recipe with HPCs as disintegrants were measured, and a decrease in the intensity of the peak derived from APAP-OXA and the appearance of the peak derived from APAP were observed as the granulation progressed (Fig. 4b). To quantitatively analyze cocrystal dissociation, the obtained LF-Raman spectra were subjected to multivariate analysis. The results of MCR analysis showed that two principal components, APAP (APAP (PC-1) and APAPOXA (PC-2)), could be detected through wet granulation (Fig. $4 \mathrm{c})$. The peak intensities derived from APAP-OXA and APAP gradually decreased and increased, respectively, with granula- 

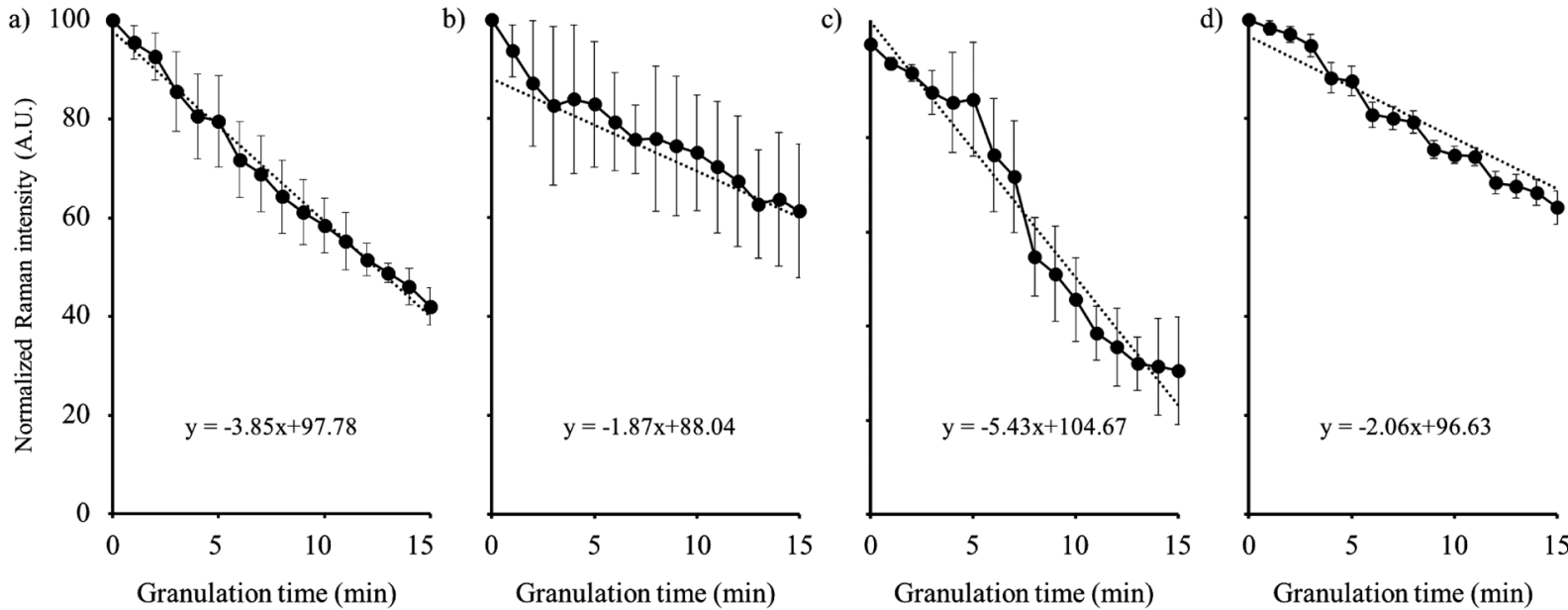

Fig. 5. Transition of Normalized Raman Intensity of Each Cocrystal during Granulating Processes by Multivariate Curve Resolution Analysis of a) APAP-OXA, b) APAP-MLA, c) APAP-TMG, and d) APAP-TOSA

The slopes of the fitted curve indicated the dissociation rate of each crystalline complex containing APAP. $(n=3$, Mean \pm standard deviation (S.D.))

tion time (Fig. 4d). Thus, MCR analysis of LF-Raman spectra indicated that APAP-OXA dissociation progressed gradually during wet granulation, resulting in the formation of APAP. While Otaki et al. ${ }^{27)}$ monitored the dissociation of cocrystals in fluidized bed granulation by LF-Raman, the present study revealed that LF-Raman can monitor the dissociation behavior of APAP-OXA during the wet granulation process in detail. Additionally, we succeeded in detailed monitoring the dissociation of only $5 \%$ cocrystals, while Nomura et al. ${ }^{32)}$ monitored cocrystal dissociation in granulation formulations containing $35 \%$ cocrystals. This is due to the use of the Raman spectrometer with a shorter laser wavelength and higher laser power compared to the Raman spectrometer used previously. ${ }^{32)}$ In general, Raman spectral measurements with a shorter laser wavelength and higher laser power can increase Raman intensity, reduce spectral noise, and shorten spectral acquisition time. ${ }^{22,37)}$ Therefore, in this study, the dissociation behavior of cocrystals with low additive amounts could be monitored in real time.

Comparison of the Dissociation Rates of Cocrystals in Wet Granulation In the screening of cocrystals, coformers must be selected carefully after considering the results of various studies because coformers affect physicochemical properties, such as the solubility of formed cocrystals. Stability is one of the properties that should be carefully considered because it significantly impacts the quality of final products. To reveal the effect of the different coformers on the stability of cocrystals containing APAP during wet granulation process, we calculated the dissociation rate of cocrystals using the MCR analysis of LF-Raman spectra obtained from the monitoring of granulation using each cocrystal. Additionally, we detected the coformer that provided the relatively most stable cocrystal. Furthermore, we also evaluated the amide salt consisting of APAP and TOSA because a difference in stability between cocrystals and amide salts was expected due to the differences in intermolecular interactions. MCR analysis suggested that the dissociation of the crystalline complexes gradually progressed during wet granulation in both cocrystals and amide salts (Figure S3-S5). The dissociation rates of APAPOXA, APAP-MLA, APAP-TMG, and APAP-TOSA were 3.85, 1.87, 5.43, and 2.06 A.U./min, respectively (Fig. 5). These results suggested that the stability of these crystalline complexes was high in the order of APAP-MLA, APAP-TOSA, APAP-OXA and APAP-TMG. This order of dissociation rate was consistent with the results of PXRD screening, suggesting that LF-Raman can be used to compare the stability of different cocrystals in coformers. Additionally, the dissociation rate of APAP-TOSA was almost the same as that of APAP-MLA. We previously reported that the amide salt was relatively more stable than the cocrystals because the cocrystal dissociated to form APAP-TOSA when the physical mixture of APAPOXA and TOSA or APAP-MLA and TOSA was ground. ${ }^{38)}$ As mentioned above, amide salts are formed through ionic bonds, and cocrystals are formed through nonionic intermolecular interactions, such as hydrogen bonds. Under the present wet granulation conditions, the stability of APAP-TOSA was higher than that of APAP-TMG and APAP-OXA, suggesting that APAP-TOSA has the same stability as APAP-MLA. These results revealed that APAP-MLA was the most stable among the cocrystal investigated in this study and monitoring the dissociation of crystalline complexes during wet granulation by LF-Raman could relatively compare the stability of crystalline complexes with different counter materials.

Effect of Disintegrants on the Dissociation of APAPOXA Pharmaceutical additives are used to provide the final products with various pharmaceutical properties. Disintegrants affect the in vivo disintegration, bioavailability, and usability of solid dosage forms. Recently, disintegrants have been added to orally disintegrating (OD) tablets, which are easier for administration to the elderly and patients who have difficulty swallowing. Disintegrants are classified as wicking type or swelling type according to the difference in disintegration mechanism, and disintegrants have varying molecular structures. ${ }^{39)}$ Onuki et $a{ }^{40)}$ analyzed the relaxation time, water absorption ratio, and disintegration time of tablets containing disintegrants using self-organizing maps (SOMs) and reported the effects of disintegration type on water behavior in the formulation. Therefore, it was expected that the disintegrants affecting the water behavior in the formulation would also affect cocrystal dissociation when cocrystals were selected as the solid-state form. The dissociation rate of APAP-OXA in granulation formulations with disintegrants shown in Table 1 
was calculated to evaluate the effect of the disintegrants on the dissociation behavior of APAP-OXA in the wet granulation process. The dissociation rate of APAP-OXA with HPC and CSW was similar to that without disintegrants and higher than that with CMC and CPR (Fig. 6). This result indicated that the addition of HPC and CSW to the granulation recipe had no effect on the dissociation behavior of APAP-OXA. Additionally, the dissociation rate of APAP-OXA with CRP was slower than that with CMC. It has been reported that the dissociation reaction of cocrystals in grinding with disintegrants was induced by process-induced lattice disorder at the boundary between cocrystals and water localized on the surface of disintegrant particles. ${ }^{30)}$ Therefore, it is considered that the water behavior in the granulation recipe contributes significantly to cocrystal dissociation. The disintegrants used in this study, except for CRP, were classified as swelling types, and the order of the water absorption ratio of swelling-type disintegrants (CMC, HPCw, HPCs and CSW were higher in that order) ${ }^{40)}$ was consistent with the order of the dissociation rate of APAP-OXA with the corresponding disintegrants. Swellingtype disintegrants cause tablet disintegration by water absorption, swelling, and gelation. The dissociation rate of APAPOXA was the lowest when CMC, which had the highest water absorption among the swelling-type disintegrants, was added. Alternatively, the dissociation rate was higher when CSW and $\mathrm{HPC}$, which have low water absorption, were added than when $\mathrm{CMC}$ was added. $\mathrm{CMC}$, which has a high water absorption ratio, can swell by absorbing a large amount of water into

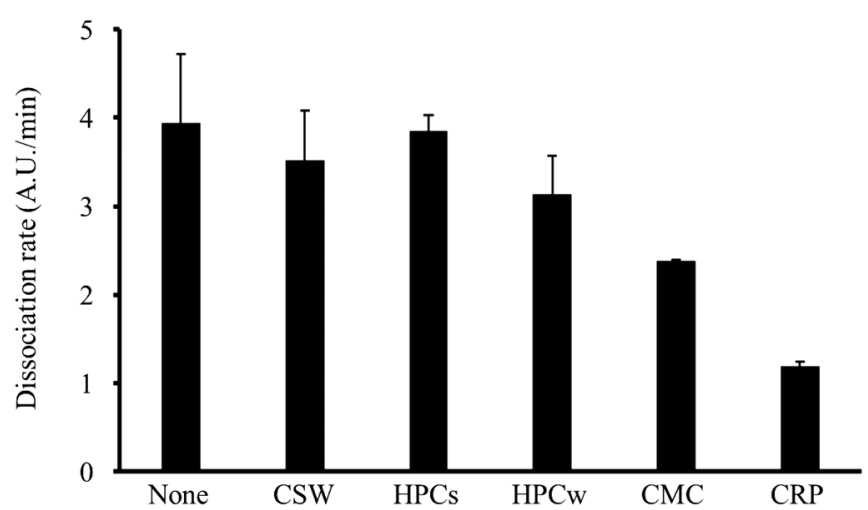

Fig. 6. Effect of the Addition of Disintegrants on the Dissociation Rate of APAP-OXA

$(n=3$, Mean \pm S.D. $)$ the particles, while CSW and HPC have low water absorption ratios and can swell with a small amount of water, resulting in an increase in the surface area of the particles. Additionally, swelling-type disintegrants form a gel layer on their particle surface after water absorption, making it difficult for water to penetrate disintegrant particles. ${ }^{41)}$ Therefore, it is expected that the water content in the bulk content will increase. From these results, we suggested that swelling-type disintegrants (CSW and HPC) with low water absorption absorbed a small amount of water, and the gel layer that formed on the surface of the disintegrants prevented the further absorption of water into the disintegrants. Consequently, the remaining water in the bulk content promoted the dissociation of APAP-OXA. In contrast, the swelling-type disintegrant (CMC) with a high water absorption rate formed a gel layer on the surface of the disintegrant after absorbing a large amount of water; thus, the amount of water remaining in the bulk content was relatively small, and the dissociation rate of APAP-OXA was considered to be slower than that of CSW and HPC. These results suggested that the water absorption ratio of disintegrants was related to the dissociation rate of APAP-OXA when swellingtype disintegrants were added. On the other hand, wickingtype disintegrants promote initial tablet disintegration by conducting water into the void in the tablet. ${ }^{39}$ In particular, CRP has a porous structure with a large specific surface area, which allows for water to quickly penetrate disintegrant particles within $7 \mathrm{~s} .{ }^{41,42)}$ In addition, Swelling-type disintegrants take 1-2 min to swell and then gel, while wicking-type disintegrants only swells slightly after water absorption. ${ }^{43)}$ These reports suggested that the water absorption behavior of CRP and the other disintegrants used in this study is significantly different, and that the water behavior in the granulation formulation using CRP is significantly different from that of the swelling-type. Therefore, APAP-OXA dissociation on the disintegrant surface was less likely to occur with CRP than with a swelling-type disintegrant because the water was absorbed inside the disintegrant, and the amount of water on the surface of the disintegrant was low (Fig. 7). In conclusion, we found that the type of disintegrant affected the dissociation rate of APAP-OXA during wet granulation, which was due to the difference in the water behavior on the disintegrant surface. It was suggested that CRP was the most effective disintegrant that could not affect the dissociation of APAP-OXA in this study. These findings suggested that monitoring the solid-state forms during the manufacturing process by LF-Raman is a

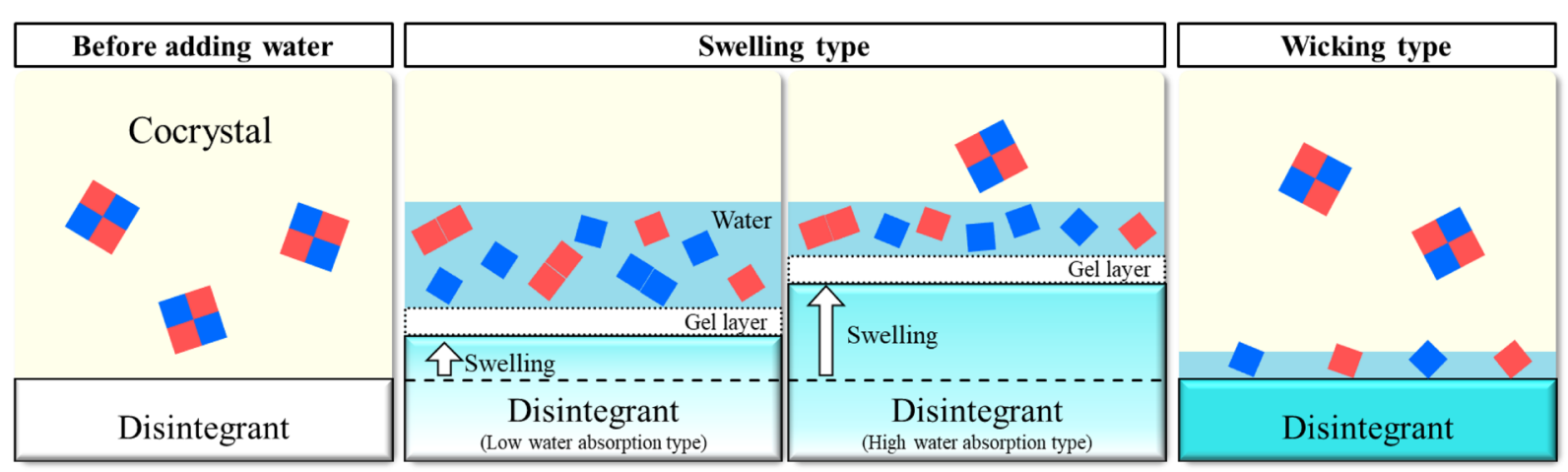

Fig. 7. Scheme of the Disintegration Mechanism of Cocrystals Due to Different Disintegrants

(Color figure can be accessed in the online version.) 
beneficial method for selecting stable cocrystals in the wet granulation process.

\section{Conclusion}

In the development of pharmaceuticals containing cocrystals as the solid-state form, selecting an optimal coformer is essential for the design of high-quality drugs. Particularly, stability is one of the criteria for selecting the solid-state form, and it is necessary to carefully evaluate the presence or absence of cocrystal dissociation from the early stage of development. In the present study, to evaluate the effect of countermaterials and disintegrant added formulation as excipients on the relative stability of crystalline complexes in the wet granulation process, the dissociation of the complexes was monitored by Raman spectrometer with high laser power and short laser wavelength, and the dissociation rate was calculated and compared. The monitoring of wet granulation of the formulation containing APAP-OXA by LF-Raman spectroscopy over time revealed that APAP-OXA gradually dissociated with water addition. Additionally, the dissociation rates of the three cocrystals containing APAP with different coformers were different from each other. These differences in dissociation rate were consistent with the order of dissociability of cocrystals obtained by PXRD, suggesting that this method using LFRaman is useful for selecting cocrystals with high stability. Furthermore, it was found that the disintegration mechanism of disintegrants and the difference in water absorption of disintegrants affected the dissociation rate of APAP-OXA, and the dissociation rate of APAP-OXA was slowest when CRP was used. From these results, the monitoring of cocrystals in the manufacturing process by LF-Raman is a useful tool to evaluate relative stability of cocrystal in real time. These findings will be expected to be helpful in the development and continuous manufacturing of cocrystal-based pharmaceuticals, which are expected to become more widespread in the future.

Acknowledgments We are grateful to Shin-Etsu Chemical Co., Ltd. (L-HPC ${ }^{\circledR}$ LH-21, NBD-21), BASF Japan (Kollidon ${ }^{\circledR}$ CL-F), Asahi Kasei Corp. (KICCOLATE ${ }^{\mathrm{TM}} \mathrm{NS}-2 \mathrm{HS}$ ) and Nihon Shokuhin Kako Co., Ltd. (Cornstarch W) for supplying additives. This work was supported by a JSPS Grantin-Aid for Scientific Research (C) Grant Number JP17K08253.

Conflict of Interest The authors declare no conflict of interest.

Supplementary Materials The online version of this article contains supplementary materials.

\section{References}

1) Chen Y., Li L., Yao J., Ma Y. Y., Chen J. M., Lu T. B., Cryst. Growth Des., 16, 2923-2930 (2016).

2) Shimpi M. R., Alhayali A., Cavanagh K. L., Rodríguez-Hornedo N., Velaga S. P., Cryst. Growth Des., 18, 4378-4387 (2018).

3) FDA. "Regulatory classification of pharmaceutical co-crystals guidance for industry," 2018, pp. 1-24.

4) EMA. "Reflection paper on the use of cocrystals of active substances in medicinal products," 2015, pp. 1-10.

5) Novartis AG Home Page, 〈https://www.novartis.com/news/mediareleases/novartis-new-heart-failure-medicine-lcz696-now-called-entrestotm-approved-fda-reduce-risk-cardiovascular-death-and-heartfailure-hospitalization>, cited 7 July, 2015
6) Astellas Pharma Inc, Home Page.: 〈https://www.astellas.com/jp/en/ news/11351>, cieted 17 January, 2014.

7) Boksa K., Otte A., Pinal R., J. Pharm. Sci., 103, 2904-2910 (2014).

8) Korde S., Pagire S., Pan H., Seaton C., Kelly A., Chen Y., Wang Q., Coates P., Paradkar A., Cryst. Growth Des., 18, 2297-2304 (2018).

9) Chavan R. B., Thipparaboina R., Yadav B., Shastri N. R., Drug Delivery Transl. Res., 8, 1726-1739 (2018).

10) Mascia S., Heider P. L., Zhang H., Lakerveld R., Benyahia B., Barton P. I., Braatz R. D., Cooney C. L., Evans J. M. B., Jamison T. F., Jensen K. F., Myerson A. S., Trout B. L., Angew. Chem., 125, 12585-12589 (2013).

11) Lee S. L., O’Connor T. F., Yang X., Cruz C. N., Chatterjee S., Madurawe R. D., Moore C. M. V., Yu L. X., Woodcock J., J. Pharm. Innov., 10, 191-199 (2015).

12) FDA. "Quality considerations for continuous manufacturing guidance for industry-draft guidance," 2019, pp. 1-23.

13) Aaltonen J., Kogermann K., Strachan C. J., Rantanen J., Chem. Eng. Sci., 62, 408-415 (2007).

14) Heinz A., Savolainen M., Rades T., Strachan C. J., Eur. J. Pharm. Sci., 32, 182-192 (2007).

15) Hisada H., Okayama A., Hoshino T., Carriere J., Koide T., Yamamoto Y., Fukami T., Chem. Pharm. Bull., 68, 155-160 (2020).

16) Wikström H., Marsac P. J., Taylor L. S., J. Pharm. Sci., 94, 209-219 (2005).

17) Strachan C. J., Rades T., Gordon K. C., Rantanen J., J. Pharm. Pharmacol., 59, 179-192 (2007).

18) Ono T., ter Horst J. H., Jansens P. J., Cryst. Growth Des., 4, 465469 (2004).

19) Tres F., Treacher K., Booth J., Hughes L. P., Wren S. A. C., Aylott J. W., Burley J. C., J. Control. Release, 188, 53-60 (2014).

20) Sašić S., "Pharmaceutical applications of Raman spectroscopy," ed. by Šašić S., John Wiley \& Sons, London, 2007, pp. 1-28.

21) Hisada H., Inoue M., Koide T., Carriere J., Heyler R., Fukami T., Org. Process Res. Dev., 19, 1796-1798 (2015).

22) Larkin P. J., Dabros M., Sarsfield B., Chan E., Carriere J. T., Smith B. C., Appl. Spectrosc., 68, 758-776 (2014).

23) Iwata K., Karashima M., Ikeda Y., Inoue M., Fukami T., CrystEngComm, 20, 1928-1934 (2018).

24) Koide T., Fukami T., Hisada H., Inoue M., Carriere J., Heyler R. Katori N., Okuda H., Goda Y., Org. Process Res. Dev., 20, 19061910 (2016).

25) Suzuki Y., Muangnoi C., Thaweesest W., Teerawonganan P., Ratnatilaka Na Bhuket P., Titapiwatanakun V., Yoshimura-Fujii M., Sritularak B., Likhitwitayawuid K., Rojsitthisak P., Fukami T., Biol. Pharm. Bull., 42, 1004-1012 (2019).

26) Tanabe Y., Maeno Y., Ohashi K., Hisada H., Roy A., Carriere J. Heyler R., Fukami T., Eur. J. Pharm. Biopharm., 136, 131-137 (2019).

27) Otaki T., Tanabe Y., Kojima T., Miura M., Ikeda Y., Koide T., Fukami T., Int. J. Pharm., 542, 56-65 (2018)

28) Inoue M., Hisada H., Koide T., Carriere J., Heyler R., Fukami T., Org. Process Res. Dev., 21, 262-265 (2017).

29) Inoue M., Hisada H., Koide T., Carriere J., Heyler R., Fukami T., Ind. Eng. Chem. Res., 56, 12693-12697 (2017).

30) Kaur N., Duggirala N. K., Thakral S., Suryanarayanan R., Mol. Pharm., 16, 3167-3177 (2019).

31) Duggirala N. K., Vyas A., Krzyzaniak J. F., Arora K. K., Suryanarayanan R., Mol. Pharm., 14, 3879-3887 (2017).

32) Nomura K., Titapiwatanakun V., Hisada H., Koide T., Fukami T., Eur. J. Pharm. Biopharm., 147, 1-9 (2020).

33) Fukui K., Suzuki Y., Haneishi K., Titapiwatanakun V., Hisada H., Koide T., Fukami T., Thai Journal of Phamaceutical Sciences, in press.

34) Good D. J., Rodríguez-Hornedo N. R., Cryst. Growth Des., 9, 2252-2264 (2009)

35) Maeno Y., Fukami T., Kawahata M., Yamaguchi K., Tagami T., 
Ozeki T., Suzuki T., Tomono K., Int. J. Pharm., 473, 179-186 (2014).

36) Suzuki N., Kawahata M., Yamaguchi K., Suzuki T., Tomono K., Fukami T., Drug Dev. Ind. Pharm., 44, 582-589 (2017).

37) Kim M., Chung H., Woo Y., Kemper M., Anal. Chim. Acta, 579 209-216 (2006).

38) Suzuki N., Kanazawa T., Takatori K., Suzuki T., Fukami T., Cryst. Growth Des., 20, 590-599 (2019).

39) Desai P. M., Liew C. V., Heng P. W. S., J. Pharm. Sci., 105, $2545-$ 2555 (2016).
40) Onuki Y., Kosugi A., Hamaguchi M., Marumo Y., Kumada S., Hirai D., Ikeda J., Hayashi Y., J. Drug Delivery Sci. Technol., 43, 141-148 (2018).

41) Yang B., Wei C., Yang Y., Wang Q., Li S., Drug Dev. Ind. Pharm., 44, 1417-1425 (2018)

42) Katsuno Y., Kishi J., J. Jpn. Soc. Pharm. Mach. Eng., 21, 149-154 (2012). ISSN: 2186-3237

43) Rojas S. G. J., Guisao S., Ruge V., AAPS PharmSciTech, 13, 10541062 (2012) 